\title{
An Interesting Case of Misdiagnosed Inflammatory Bowel Disease With Immunoproliferative Small Intestine Disorder
}

\author{
Neda Nozari ${ }^{1,}$; Homayoon Vahedi ${ }^{1}$; Amir Reza Radmard ${ }^{1}$; Masoud Sotoudeh ${ }^{1}$; Parisa \\ Divsalar $^{2}$ \\ ${ }^{1}$ Digestive Diseases Research Center, Shariati Hospital, Tehran University of Medical Sciences, Tehran, IR Iran \\ ${ }^{2}$ The Priory Hospital Cheadle Royal, Cheadle, Cheshire, Manchester, UK \\ ${ }^{*}$ Corresponding Author: Neda Nozari, Digestive Diseases Research Center, Shariati Hospital, Tehran University of Medical Sciences, Tehran, IR Iran. Tel: +98-2182415000, Fax: +98- \\ 2182415400, E-mail: nozari_neda@yahoo.com
}

Received: May 15, 2013; Revised: August 10, 2013; Accepted: May 1, 2014

\begin{abstract}
Introduction: Inflammatory bowel disease(IBD) prevalence is increasing in Asia. Clinicians are faced with misdiagnosis for IBD even with classic symptoms. This case report shows that how a delayed diagnosis of IBD can produce significant problems for patient.

Case Presentation: A 45 year-old man and known case of IPSID (Immunoproliferative small intestine disorder) was referred for follow-up to our GI (Gastrointestinal) clinic. He presented with a history of intractable watery diarrhea along with slight weight loss for the last 22 years. He was admitted with an episode of severe abdominal pain and acute peritonitis 10 years ago. At the time, laparotomy was performed due to a suspicious appendicular mass; however, the surgeon discovered a cecum mass and extended adhesion during laparotomy. According to IPSID diagnosis, he was then referred to an oncologist who treated him with chemotherapy and patient was advised to attend an annual colonoscopy checkup as follow-up. He was gradually complicated by renal stones, arthritis, hypoalbuminemia and bone disease during the follow-up. We as gastroenterologists reviewed his history again and requested a revision for all histopathology results by GI pathologist. Pathologist confirmed IBD (Crohn's disease) from the first pathology and his treatment was started immediately.

Discussion: Several diseases can mimic IBD symptoms but with careful consideration of patient's symptoms, endoscopic findings and pathology results one should be able to diagnose IBD. Correct diagnosis of IBD can affect the quality of life of patient.
\end{abstract}

Keywords:Inflammatory Bowel Diseases; Diagnostic Errors; Intestine, Small

\section{Introduction}

The prevalence of inflammatory bowel disease (IBD) in Asia is lower than western countries. Nowadays, clinicians have yet faced with misdiagnosis of IBD even with classical symptoms including abdominal pain, diarrhea and rectal bleeding. Any delay in diagnosis of IBD can produce significant problems for patient. Astonishingly, patients with IBD are diagnosed with delay in the presence of advanced diagnostic tools.

\section{Case Presentation}

A 45 year-old man and known case of IPSID (Immunoproliferative small intestine disorder) was referred for follow-up to our GI (gastrointestinal) clinic. He presented with a 22-year history of intractable watery diarrhea along with slight weight loss. Chronic diarrhea had gradual onset, most of times, as often as 6 to 10 times a day and three times in the recent months. He never noticed pus or mucus in his stools. Nevertheless, 16 years ago, he had reported a slight fresh rectal bleeding in early phase of disease for at least a few months when hemor- rhoidectomy and perianal fistulectomy were performed. Consequently, rectal bleeding improved completely but diarrhea was continued. This was relatively a painless condition. The patient underwent his first colonoscopy 13 years ago when just a solitary ulcer in descending colon was seen. Three years later, he was admitted to a hospital with a history of severe abdominal pain along with distension. Physical examination revealed a moderately tender definite mass at the right lower quadrant of his abdomen.

\subsection{Ultrasonography Reported}

A mass $(50 \times 70 \mathrm{~mm})$ was found at his right lower quadrant suggestive of abscess. Therefore, laparotomy was performed regarding a suspicious appendicular mass. However, surgeon discovered a cecum mass and extended adhesion during laparotomy. According to the diagnosis of cecum tumor (IPSID), right hemicolectomy was performed which was confirmed by a pathologist. As a result, he was referred to an oncologist and received chemothera- 
py for six sessions. After chemotherapy, he was under close follow-up with colonoscopy each year and biopsy had been obtained at every session. In each follow-up session, he was introduced with IPSID diagnosis to gastroenterologist and pathologist by oncologist. Pathologist reported some infiltration of inflammatory and lymphocyte cells and confirmed IPSID again. Although alkaline phosphates gradually increased, alpha heavy chain had negative results in several times. Two years later, he was diagnosed with bilateral renal stones and received medical therapy. In addition, he had to do transurethral lithotomy due to hydronephrosis and multiple bilateral renal stones. One year later, he had arthritis in his left foot for a few days, which his joint pain was subsided after a week. Laboratory data reported normal ANA (anti-nuclear antibody), Complement 3, 4, 50, Anti CCP (Antibodies to cyclic citrullinated polypeptides), and RF (Rheumatic factor). Wright and coombs tests had negative results. Although he was gradually complicated by diarrhea, clinicians did not consider any diagnosis for his condition except IPSID and then he just was followed by supportive medicine.

On physical examination, the patient was thin, weak, depressed and pale. Vital signs were as follows: Blood Pressure: 118/72 mmHg; Pulse Rate: 74/min; Respiratory Rate: 16/min; Temperature: $37^{\circ} \mathrm{C}$. A midline laparotomy scar was seen in abdominal examination. Rectal examination revealed no masses, partially formed stools and no localized tenderness. Sarcopenia was present, but temporal wasting was not significant. Other examinations had normal findings. We as gastroenterologists reviewed his history carefully this time and requested a revision of all his histopathology results by GI pathologist to conclude IBD. Pathologist revised them and confirmed IBD (CD, Crohn's disease) from the first pathology (Figure 1). Magnetic resonance (MR) enterography showed multiple strictures in anastomosis site (ileocolic), in transverse and left parts of colon (Figure 2).

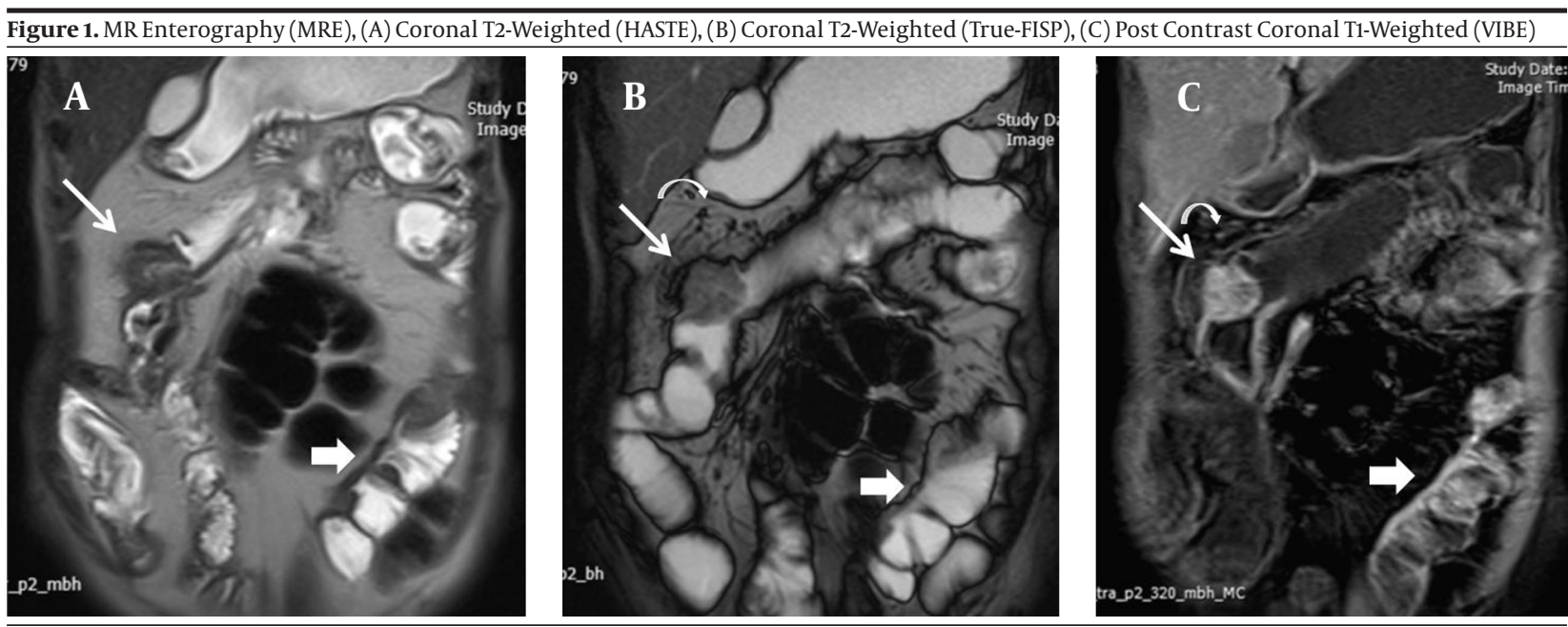

There is considerable mural thickening with mass like appearance at ileocolic anastomotic area with intense contrast enhancement leading to luminal stricture (thin arrow). Adjacent mesenteric enlarged lymph nodes are also depicted (curved arrow). Another short segment mural thickening is evident in mid ileum with predominant involvement of mesenteric side showing increased contrast enhancement (thick arrow).

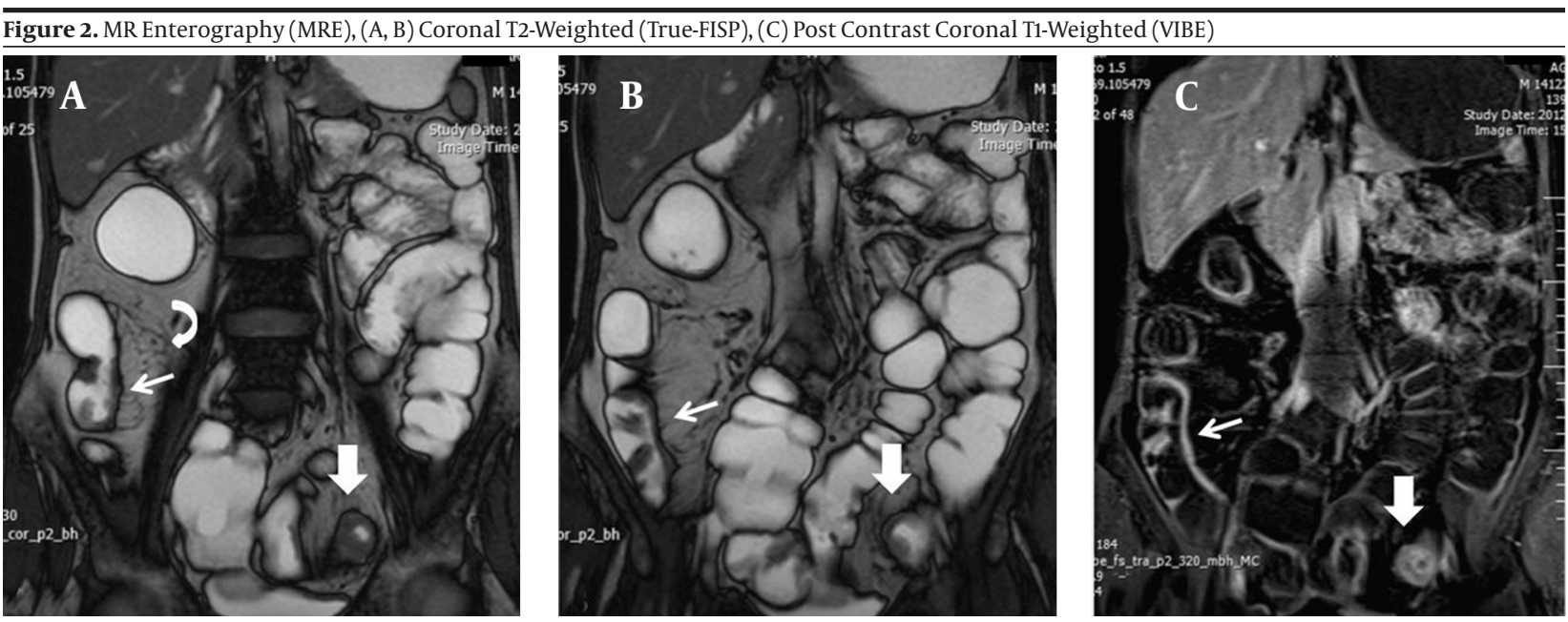

A focal short segment mural thickening is seen in distal ileum mostly mesenteric border with marked contrast enhancement (thin arrow), which is accompanied with adjacent increased mesenteric vascularity. There is also circumferential mural thickening of sigmoid, which shows typical target appearance in post contrast image in favor of active Crohn's disease (thick arrow). 
Multiple biopsies were taken from stenotic lesion in descending colon through flexible sigmoidoscopy, but pathology did not show any malignant cell. Last laboratory data were as follows: Creatinine: $1 \mathrm{ng} / \mathrm{mL}$ (GFR: 66 mL/min, MDRD: $85.6 \mathrm{~mL} / \mathrm{min})$, AST: $20 \mathrm{IU} / \mathrm{L}$, ALT: $13 \mathrm{IU} / \mathrm{L}$, Total Bilirubin: $0.7 \mathrm{~g} / \mathrm{dL}$, Direct Bilirubin: $0.1 \mathrm{gr} / \mathrm{dL}, \mathrm{LDH}$ : 296 IU/L, WBC: 10600/mm³ $\mathrm{mB}^{3}$ g g/dL, Ferritin: $5 \mu \mathrm{g} / \mathrm{L}$, PLT: 422000/ $\mathrm{mm}^{3}$, Albumin: $1.8 \mathrm{~g} / \mathrm{dL}$, ESR: $21 \mathrm{~mm}$ 1st hour, ALP: 396 IU/L (350), Gamma glutamyl transpeptidase: 26 U/L (normal), CA 19-9: $8.8 \mathrm{U} / \mathrm{mL}$ (normal), CEA: $3.9 \mathrm{ng} / \mathrm{mL}$ (normal). Urine analysis showed excretion of moderate calcium oxalate and proteinuria (250 mg/24 hours). Prednisolone $40 \mathrm{mg}$ daily, Mesalamine $4 \mathrm{~g}$ daily, Folic acid 5 $\mathrm{mg}$ daily and Ca-D twice daily were ordered immediately. His general condition improved after 1 month and then corticosteroid was tapered and replaced with Azathioprine $100 \mathrm{mg}$ daily later in a few months.

\section{Discussion}

Inflammatory bowel disease (IBD) has increased in Asia and related challenges with diagnosis of IBD are also increasing (1). IBD may present in a variety of ways including abdominal pain, weight loss, diarrhea and rectal bleeding. Clinicians should provide an elaborative history and data to pathologist to reach a true diagnosis; otherwise, it could easily lead to a misdiagnosis of IBD. Crohn's disease can present $10 \%-30 \%$ with abdominal and pelvic abscess during the life time of disease (2). Appendicular involvement in Crohn's disease is common (21\%). Sonography is a useful detector for differentiating between Crohn's disease and appendix involvement and acute appendicitis (3). Resection of the ileocaecal segment is recommended for the ileocaecal involvement (4). But right hemicolectomy was performed for appendicular mass in this case with suspicion of cecum tumor at first hospitalization.

Suspected samples of IBD and other diseases like solitary rectal ulcer syndrome should be referred to another pathologist for diagnosis confirmation (5). For example, colon primary lymphoma can be misdiagnosed with IBD (6). A study performed in Iran reported a dramatic decrease in the incidence of IPSID (nearly 3\% of all gastrointestinal cancers) due to decreasing exposure to infectious agents during 1974-1999 (7). Clinicians might mistake IBD with IPSID at the first visit without any background, but diagnosis of IBD is simple when clinician faces with this case and a long history of GI symptoms and extra-intestinal manifestations in later phase. Clinician should notice that, the prevalence of IPSID has recently decreased in Iran and the extra-intestinal manifestations and intestinal strictures are more common in IBD than IPSID disease. IBD may lead to some extra-intestinal diseases like renal stone disease and arthritis. Malabsorption and protein losing enteropathy may induce nutrients defi- ciencies in IBD. Patients with CD can present with osteoporosis or osteopenia even without corticosteroid treatment (8). This patient was complicated by bone disease in a long period of illness activity in spite of not receiving any kind of corticosteroid treatment.

Hyperoxaluria can induce calcium oxalate renal stones (8). MR enterography is the best technique in the followup of IBD and can show intra-luminal and extra-luminal bowel diseases (9). If gastroenterologists who conducted follow-ups had paid more attention to his intestinal and extra-intestinal symptoms, they would have noticed IBD and exclude IPSID easily. IBD should be taken into account for patients with extra-intestinal symptoms like this patient.

Delay in diagnosis of IBD is greater in CD than UC (ulcerative colitis). Correct diagnosis of IBD affects patients' quality of life. Pathologists should be cautious when describing "colitis" in colon biopsies. It is better that clinicians follow difficult cases to reach definite IBD diagnosis.. Several diseases may mimic IBD symptoms; however, with careful consideration of patient's symptom, endoscopic findings and pathology results we should be able to detect most of IBD patients.

\section{Authors' Contribution}

All authors participated in the study equally.

\section{Financial Disclosure}

There was no conflict of interest.

\section{References}

1. Makharia GK. Rising incidence and prevalence of Crohn's disease in Asia: is it apparent or real? J Gastroenterol Hepatol. 2006;21(6):929-31.

2. Richards RJ. Management of abdominal and pelvic abscess in Crohn's disease. World J Gastrointest Endosc. 2011;3(11):209-12.

3. Ripolles T, Martinez MJ, Morote V, Errando J. Appendiceal involvement in Crohn's disease: gray-scale sonography and color Doppler flow features. AJR Am J Roentgenol. 2006;186(4):1071-8.

4. Machado NO, Chopra PJ, Hamdani AA. Crohn's disease of the appendix with enterocutaneous fistula post-appendicectomy: An approach to management. NAm J Med Sci. 2010;2(3):158-61.

5. Derakhshani S, Pakzad M, Vafaie M, Tehrani TS, Abdollahi M. A report of 112 cases of solitary rectal ulcer syndrome from Iran. Cent EurJ Med. 2009;4(1):49-53.

6. Ramasamy KA, Vignaraja R, Hastings AG, Bessell EM, Snape J. A case of primary non-Hodgkin's lymphoma of the transverse colon presenting as inflammatory bowel disease. Eur J Gastroenterol Hepatol. 2002;14(12):1401-3.

7. Lankarani KB, Masoompour SM, Masoompour MB, Malekzadeh R, Tabei SZ, Haghshenas M. Changing epidemiology of IPSID in Southern Iran. Gut. 2005;54(2):311-2.

8. Jose FA, Heyman MB. Extraintestinal manifestations of inflammatory bowel disease. J Pediatr Gastroenterol Nutr. 2008;46(2):124-33.

9. Tolan DJ, Greenhalgh R, Zealley IA, Halligan S, Taylor SA. MR enterographic manifestations of small bowel Crohn disease. Radiographics. 2010;30(2):367-84. 